\title{
Theory of ground state factorization in quantum cooperative systems
}

\author{
Salvatore M. Giampaolo, ${ }^{1,2}$ Gerardo Adesso, ${ }^{1,2}$ and Fabrizio Illuminati ${ }^{1,2,3, \text {, }}$ \\ ${ }^{I}$ Dipartimento di Matematica e Informatica, Università degli Studi di Salerno, Via Ponte don Melillo, I-84084 Fisciano (SA), Italy \\ ${ }^{2}$ CNR-INFM Coherentia, Napoli, Italy; CNISM, Unità di Salerno; and INFN, \\ Sezione di Napoli - Gruppo Collegato di Salerno, Italy \\ ${ }^{3}$ ISI Foundation for Scientific Interchange, Viale Settimio Severo 65, I-10133 Turin, Italy
}

(Dated: March 31, 2008)

\begin{abstract}
We introduce a general analytic approach to the study of factorization points and factorized ground states in quantum cooperative systems. The method allows to determine rigorously existence, location, and exact form of separable ground states in a large variety of, generally non-exactly solvable, spin models belonging to different universality classes. The theory applies to translationally invariant systems, irrespective of spatial dimensionality, and for spin-spin interactions of arbitrary range.

PACS numbers: 75.10.Jm, 03.67.Mn, 73.43.Nq, 03.65.Ca
\end{abstract}

Introduction.- Quantum engineering and quantum technology have been developing at a fast pace in recent years. Quantum devices are being vigorously pursued for applications ranging from nano-sciences to quantum information and entanglement-enhanced metrology [1]. Despite a large variety of possible implementations involving different physical systems, many relevant properties of such devices can be investigated in a unified setting by appropriate mappings to quantum spin models [2, 3]. Thus, control of ground state entanglement in quantum spin systems plays an important role in quantum technology applications [4]. On the other hand, knowledge of exact solutions endowed with precisely determined properties of separability or entanglement, can be of great relevance in the study of advanced models of condensed matter and cooperative systems that are in general not exactly solvable.

The occurrence of totally factorized (unentangled) ground states of quantum many-body systems was first discovered in the one-dimensional anisotropic Heisenberg model with nearest-neighbor interactions [5]. This result was later rederived and extended to two dimensions using quantum Monte Carlo numerical methods [6]. Complex quantum systems exhibiting cooperative behaviors, whose ground states are typically entangled [7], may thus admit, for some non trivial values of the Hamiltonian parameters, a ground state which is completely separable. The phenomenon of ground state factorization appears to be associated with the presence of an "entanglement phase transition" with no classical counterpart [8]; furthermore, for the purposes of quantum engineering applications that employ distributed entanglement in order to manipulate and transfer information [9], factorization points need to be exactly identified and avoided to guarantee the reliable implementation of quantum devices. Finally, for models not admitting exact general solutions, achieving knowledge of the exact ground state, even if only for the restricted nontrivial set of parameters associated to factorization, would allow (i) to prove the existence of an ordered phase and characterize it; (ii) to build variational or perturbative approximations around the exact factorized solution, that may then be used as test benchmarks for the validity and the precision of numerical algorithms and simulations. Unfortunately, to date, it has been extremely hard to go beyond the pioneering result of Kurmann et al. [5] despite the fact that, to prove total factorization, it would suffice to show the vanishing of the von Neumann entropy of entanglement, or of the linear entropy (tangle) [10]. The difficulty resides in the fact that, with few special exceptions, explicit analytic expressions for these measures of entanglement cannot be obtained. Hence, the only possibility to gain insight on factorization in systems of increasing complexity has relied so far on heuristic or numerical approaches.

In the present work we introduce a general analytic method that allows to determine exactly the existence of factorized ground states and to characterize their properties in quantum spin models defined on regular lattices, in any spatial dimension, and with spin-spin interactions of arbitrary range. In correspondence to rigorously established ground state factorizability, the method also allows to determine novel sets of exact solutions in generally non exactly solvable models. Previous particular findings for models with short range interactions are rigorously re-derived and extended within a unified framework inspired by concepts of quantum information science. The method is built on a formalism of single-spin, or single-qubit, unitary operations (SQUOs) and associated entanglement excitation energies (EXEs), previously introduced for the characterization and quantification of entanglement in systems of quantum information [11, 12]. The novel techniques exploit the fundamental property enjoyed by the EXEs, of vanishing if and only if a pure state is fully factorized [12]. For any given Hamiltonian, the strategy to the understanding of factorization is first to assume as working point a phase endowed with some kind of magnetic order. Next, by imposing the vanishing of the EXE and of the linear entropy, one derives a closed set of conditions whose solutions determine uniquely the occurrence (or the non occurrence) of factorization points at which a quantum ground state is completely disentangled. Besides the rigorous determination of novel factorization points and exact solutions of generic quantum spin models, the method allows as well to re-derive analytically the few previously known results on ground state factorization [5, 6, 13]. 
The method.- To fix ideas and notations, let us consider general, translationally invariant, exchange Hamiltonians for spin-1/2 systems on $d$-dimensional regular lattices, with spinspin interactions of arbitrary range and arbitrary anisotropic couplings. This class of Hamiltonians encompasses a very large set of models describing different spin systems and spanning several universality classes like, among others, the Ising, $\mathrm{XY}$, Heisenberg, and XYZ symmetries. The general Hamiltonian can be written in the form

$$
H=\frac{1}{2} \sum_{\underline{i}, \underline{l}} J_{x}^{r} S_{\underline{i}}^{x} S_{\underline{l}}^{x}+J_{y}^{r} S_{\underline{i}}^{y} S_{\underline{l}}^{y}+J_{z}^{r} S_{\underline{i}}^{z} S_{\underline{l}}^{z}-h \sum_{\underline{i}} S_{\underline{i}}^{z} .
$$

Here $\underline{i}$ (and similarly $\underline{l}$ ) is a $d$-dimensional index vector identifying a site in the lattice, $S_{\underline{i}}^{\alpha}(\alpha=x, y, z)$ stands for the spin- $1 / 2$ operator on site $\underline{i}, h$ is external field directed along the $z$ direction, $r=|\underline{i}-\underline{l}|$ is the distance between two lattice sites, and $J_{\alpha}^{r}$ is the spin-spin coupling along the $\alpha$ direction; translational invariance implies that it depends only on the distance $r$ between the spins. Without loss of generality, one can impose $\left|J_{x}^{r}\right| \geq\left|J_{y}^{r}\right|,\left|J_{z}^{r}\right| \forall r$. This condition guarantees that at a particular value of the external field $h=h_{c}$ the system undergoes a quantum phase transition at zero temperature: For $h<h_{c}$ the system is in an ordered phase which may, or may not, be associated to a non-vanishing order parameter corresponding to the ground-state expectation of $S_{\underline{k}}^{x}$. The order parameter $M_{x}=\left\langle S_{\underline{k}}^{x}\right\rangle$ in the case of ferromagnetic order, and $M_{x}=(-1)^{i}\left\langle S_{\underline{k}}^{x}\right\rangle$ in the anti-ferromagnetic case. In the following, we specialize to the anti-ferromagnetic case; trivial modifications are needed in the ferromagnetic case.

Single-Qubit Unitary Operations (SQUOs) are unitary transformations $U_{\underline{k}}$ that leave all spins unaffected but for an arbitrarily chosen one, say, at site $\underline{k}$, on which the SQUOs act as unitary, Hermitian, and traceless operators [11]. One can prove that there exists an element of this class, the $E x$ tremal SQUO (E-SQUO) $\bar{U}_{\underline{k}}$, such that the squared Euclidean distance between a state $|\Psi\rangle$ and its image $\bar{U}_{\underline{k}}|\Psi\rangle$ under the action of the E-SQUO coincides with the linear entropy (tangle) $\tau=2\left(1-\operatorname{Tr}\left[\rho_{k}^{2}\right]\right)$, where $\rho_{k}$ denotes the reduced state of spin $k[10,11]$. This entanglement monotone quantifies the entanglement existing in state $|\Psi\rangle$ between the single-spin block $\underline{k}$ and the remainder of the system. If $|\Psi\rangle$ is the ground state of a quantum mechanical Hamiltonian $H$, the E-SQUO is uniquely associated to the aforementioned EXE, defined as $\Delta E=\left\langle\bar{U}_{\underline{k}} H \bar{U}_{\underline{k}}\right\rangle-\langle H\rangle$. A crucial property of the EXE is that if $H$ is translationally invariant and $\left[H, U_{\underline{k}}\right] \neq 0$ for all SQUOs $U_{\underline{k}}$, then the ground state is completely factorized if and only if $\Delta E=0$ [12]. The generic spin- $1 / 2$ models Eq. (1), as well as many others, satisfy this condition. The above theorem can then be applied to identify the occurrence of factorization points. By definition, the E-SQUO can be written as $\bar{U}_{\underline{k}}=\bigotimes_{\underline{i} \neq \underline{k}} \mathbf{1}_{\underline{i}} \otimes O_{\underline{k}}$ where, following Ref. [11], $O_{\underline{k}}$ can be written as

$$
O_{\underline{k}}^{ \pm}=S_{\underline{k}}^{z} \cos \theta \pm S_{\underline{k}}^{x} \sin \theta
$$

where the \pm sign discriminates the two sublattices, corresponding to the sign of the staggered magnetization $\left\langle S_{\underline{k}}^{x}\right\rangle$.

Let us assume that $H$, Eq. (1), admits a factorized ground state. Applying the E-SQUO, Eq. (2), to the ground state, exploiting the fact that for fully factorized states all correlations separate in products of single-site expectations, and imposing the condition $\Delta E=0$, one has that factorization requires the simultaneous occurrence of

$$
\tan \theta=\frac{M_{x}}{M_{z}} ; \quad \tan \theta=\frac{\mathcal{J}_{x} M_{x}}{\mathcal{J}_{z} M_{z}-h_{f}},
$$

where $h_{f}$ is the factorizing field, i.e. the value of the external field for which ground state factorization occurs. The quantities $\mathcal{J}_{\alpha}$ are the net interactions reflecting the type of magnetic order that exists along different axes. In the antiferromagnetic case, $\mathcal{J}_{x}=\sum_{r=1}^{\infty}(-1)^{r} Z_{r} J_{x}^{r}$ and $\mathcal{J}_{z}=\sum_{r=1}^{\infty} Z_{r} J_{z}^{r}$, where $Z_{r}$ denotes the number of sites placed at distance $r$ from a given spin. Conditions (3) and the vanishing of the tangle $\tau=1-4\left(M_{x}^{2}+M_{z}^{2}\right)$ yield a closed expression for the phase $\theta$ as a function of the Hamiltonian parameters and of $h_{f}$ :

$$
\cos \theta=\frac{2 h_{f}}{\mathcal{J}_{z}-\mathcal{J}_{x}} .
$$

Eq. (4) determines, independently of the magnetizations, the form of the candidate factorized ground state $\left|\Psi_{f}\right\rangle[14]$ :

$$
\left|\Psi_{f}\right\rangle=\bigotimes_{\underline{i}}\left|\psi_{2 \underline{i}}^{+}\right\rangle\left|\psi_{2 \underline{i}+1}^{-}\right\rangle,
$$

where $\left|\psi_{\underline{k}}^{ \pm}\right\rangle$are the eigenvectors of $O_{\underline{k}}^{ \pm}$with eigenvalue $1 / 2$.

This far, we have determined the general expression that a factorized ground state must assume. We are left to establish the conditions for its existence, i.e. the conditions under which a state of the form Eq. (5) is indeed the eigenstate of $H$, Eq. (1), with the lowest energy. For each pair of spins $\underline{i}$ and $\underline{j}$ we introduce the pair Hamiltonian

$$
H_{\underline{i} \underline{j}}=J_{x}^{r} S_{\underline{i}}^{x} S_{\underline{j}}^{x}+J_{y}^{r} S_{\underline{i}}^{y} S_{\underline{j}}^{y}+J_{z}^{r} S_{\underline{z}}^{z} S_{\underline{j}}^{z}-h_{f}^{r}\left(S_{\underline{i}}^{z}+S_{\underline{j}}^{z}\right),
$$

where $h_{f}^{r}$ is defined by the relation $2 h_{f}^{r}=\cos \theta\left(J_{z}^{r}-\right.$ $(-1)^{r} J_{x}^{r}$. It is immediate to verify that by re-summing the operators $H_{\underline{i} \underline{j}}$ over all spin pairs, one reobtains Eq. (1), with $h=h_{f}$. Hence, proving that $\left|\Psi_{f}\right\rangle$ is a simultaneous eigenstate of all pair Hamiltonians $H_{\underline{\underline{j}} \boldsymbol{j}}$, implies that it is an eigenstate of the total Hamiltonian $H$ as well. Inserting the expression of $\left|\Psi_{f}\right\rangle$ in Eq. (6) yields a set of conditions that must be satisfied to ensure that $\left|\Psi_{f}\right\rangle$ is an eigenstate of every pair Hamiltonian $H_{\underline{i j}}$ :

$$
-J_{y}^{r}+\cos ^{2} \theta J_{x}^{r}+(-1)^{r} \sin ^{2} \theta J_{z}^{r}=0 \quad \forall r,
$$

where $\theta$ is given by Eq. (44. By summing over $r$, term by term, all the relations in Eq. (7), and solving for $h_{f}$, we eventually obtain the exact, general expression of the factorizing field as a function of the net interactions along the different axes:

$$
h_{f}=\frac{1}{2} \sqrt{\left(\mathcal{J}_{x}-\mathcal{J}_{z}\right)\left(\mathcal{J}_{y}-\mathcal{J}_{z}\right)} .
$$


In Eq. (8) the net interaction along the $y$ axis depends on the anti-ferromagnetic order on the $x$ axis and, hence, it is given by $\mathcal{J}_{y}=\sum(-1)^{r} Z_{r} J_{y}^{r}$. We remark that Eq. (8) is completely general and holds for lattices of arbitrary spatial dimension and for interactions of arbitrary range.

We are left to determine the conditions under which the factorized eigenstate $\left|\Psi_{f}\right\rangle$ is associated to the lowest energy eigenvalue. A general sufficient condition is that every twosite reduced state, obtained from $\left|\Psi_{f}\right\rangle$ by a partial trace over all sites except the pair $\{\underline{i}, \underline{j}\}$ (which is still a pure state since $\left|\Psi_{f}\right\rangle$ is factorized), is the ground state of $H_{\underline{i j}}$, for every pair $\{\underline{i}, j\}$. Given the relation existing between all the pair Hamiltonians $H_{\underline{i} \underline{j}}$ and the total Hamiltonian $H$, it follows that if a state is associated to the lowest eigenvalue of every $H_{\underline{i} \underline{j}}$, then it is associated to the lowest eigenvalue of $H$. To proceed, we need to distinguish between the various possible cases, depending on the structure of the couplings $J_{\alpha}^{r}$.

Models with short range interactions.- By short range, or nearest-neighbor, we mean $J_{\alpha}^{r}=0$ for all $\alpha$ and $r \geq 2$. Anti-ferromagnetic order is ensured by having $J_{x}^{1}=1$ and $\left|J_{y, z}^{1}\right| \leq 1$. For one-dimensional models, we have $Z_{1}=2$, $\mathcal{J}_{x}=-2, \mathcal{J}_{y}=-2 J_{y}^{1}$, and $\mathcal{J}_{z}=2 J_{z}^{1}$. Inserting these quantities in Eqs. (8, 4, 5) we obtain the expressions for $\theta$ and for the factorized ground state $\left|\Psi_{f}\right\rangle$. The explicit expression of the factorizing field is $h_{f}^{(d=1)}=\sqrt{\left(1+J_{z}^{1}\right)\left(J_{y}^{1}+J_{z}^{1}\right)}$ and the energy per site reads $\varepsilon^{(d=1)}=(1 / 8)\left(\mathcal{J}_{z}-\mathcal{J}_{y}+2\right)=$ $(1 / 4)\left(1+J_{z}^{1}+J_{y}^{1}\right)$. Besides reproducing the original results of Kurmann et al. [5], the analytic method allows to establish that ground state factorization occurs for a much larger range of values of the couplings. Our general framework singles out novel instances of classical-like ground states already in this simple model, as pictorially sketched in Fig. 1.

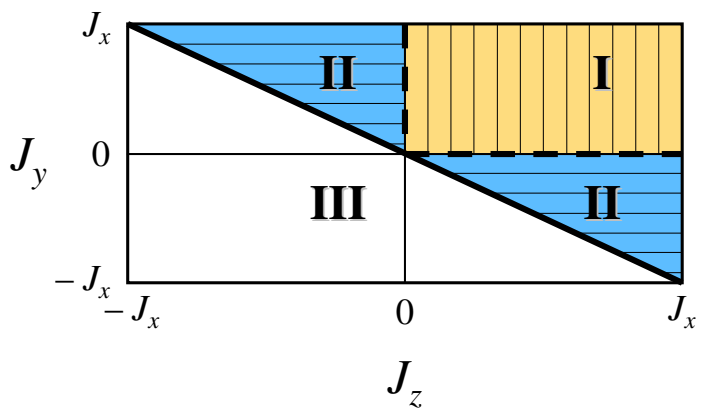

FIG. 1: (Color online) Spin-1/2 models with short range interactions: Domains in the space of couplings. Region I (vertical lines): Domain of parameters for which ground state factorization was originally identified [5], and is established rigorously in the present paper. Region II (horizontal lines): Domain in which factorization is established for the first time, and rigorously, in the present paper. Region III (no lines): Domain in which factorization is rigorously excluded. All plotted quantities are dimensionless.

The analytic approach can be extended immediately to higher-dimensional lattices. For a square lattice, the number of nearest neighbors is $Z_{1}=4$, and hence the net interactions $\operatorname{read} \mathcal{J}_{x}=-4, \mathcal{J}_{y}=-4 J_{y}^{1}$, and $\mathcal{J}_{z}=4 J_{z}^{1}$. Therefore, moving from one to two dimensions, we find that factorization occurs in the same domains of couplings and for the same value of $\theta$, but at a factorizing field and with an energy per site that are twice the corresponding quantities in one dimension: $h_{f}^{(d=2)}=2 h_{f}^{(d=1)}$ and $\varepsilon^{(d=2)}=2 \varepsilon^{(d=1)}$. These exact results recover, confirm, and extend recent numerical findings [6].

As the resources for numerical simulations scale with the lattice dimension, and in the absence of analytic approaches, it is not surprising that no study of factorization in threedimensional systems was attempted so far. However, exploiting our novel analytic method, such a study can be carried out exactly and straightforwardly. Namely, moving from square to cubic lattices we only need to insert the correct value of the coordination number $Z_{1}=6$. It is then straightforward to prove that for three-dimensional models ground state factorization occurs at the same values of the couplings as in one dimension, but at a factorization field and with an energy per site that are three times the corresponding quantities in one dimension: $h_{f}^{(d=3)}=3 h_{f}^{(d=1)}$ and $\varepsilon^{(d=3)}=3 \varepsilon^{(d=1)}$.

The ferromagnetic counterparts of the anti-ferromagnetic models can be immediately recovered performing $\pi / 2$ rotation around the $z$ axis at each lattice site in either one of the two sublattices. Hence, given a set of couplings $\left(1, J_{y}^{1}, J_{z}^{1}\right)$ for which an anti-ferromagnetic factorized ground state occurs, there exists a corresponding set of couplings $\left(-1,-J_{y}^{1}, J_{z}^{1}\right)$ for which a ferromagnetic factorized ground state occurs at the same value of the factorizing field $h_{f}$, the same energy per site, and the same value of $\theta$.

Models with finite range interactions.- In the case of spin systems with short range interactions, the set of equations Eq. (7) is automatically verified by any model that admits a real solution for Eq. (4) and Eq. (8) [15]. This redundancy is removed as soon as one considers interactions of longer spatial range, because in these cases the net interactions do not depend on the nearest-neighbor couplings alone. To illustrate this important point, let us consider ferromagnetic models in arbitrary spatial dimensions and with non-vanishing interactions up to a certain finite distance $s: J_{x, y}^{r}<0 \forall r \leq s$, $J_{x, y}^{r}=0 \forall r \geq s$, and $J_{z}^{r}=0 \forall r$. It is straightforward to verify that a sufficient condition for ground state factorization is that the ratio of the non vanishing couplings must satisfy the relation $J_{x}^{r} / J_{x}^{1}=J_{y}^{r} / J_{y}^{1} \equiv \gamma_{r} \forall r \leq s$. Otherwise, Eqs. (7) do not admit solutions. If this condition is satisfied, we have that the net interactions (that in the ferromagnetic instance read $\mathcal{J}_{x, y}=\sum_{r} Z_{r} J_{x, y}^{r}$ ) are $\mathcal{J}_{x, y}=\Gamma J_{x, y}^{1}$, where $\Gamma=\sum_{r=1}^{s} Z_{r} \gamma_{r}$. Ground state factorization occurs at $h_{f}^{(s)}=$ $\Gamma\left(J_{x}^{1} J_{y}^{1}\right)^{1 / 2}$, with an energy per site $\varepsilon=\left[\Gamma\left(J_{x}^{1}+J_{y}^{1}\right)\right] / 8$. Therefore, at exactly defined ratios of the couplings, systems with finite range interactions admit fully separable ground states analogous to the ones arising in the case of systems with only nearest-neighbor couplings. The factorizing field and the energy per site are increased exactly by a factor $\Gamma / 2$ with respect to the case of models with short range interactions. 
Models with infinite range interactions.- A very interesting limiting case is given by models with infinite range interactions, such as the fully connected or Lipkin-Meshkov-Glick (LMG) model [16], which is obtained in the limit of diverging $s$ and couplings of the form $J_{x, y}^{r}=J_{x, y}=2 \Delta_{x, y} / N \forall r$. The scaling with the number of lattice sites $N$ ensures that the net interactions converge to a finite value in the thermodynamic limit: $\mathcal{J}_{x, y} \rightarrow 2 \Delta_{x, y}$. It is then rather straightforward to solve Eqs. (7) exactly and prove rigorously that the ground state of the LMG model is a fully factorized ferromagnetic state for $\Delta_{x}=1 ; 0 \leq \Delta_{y} \leq 1 ; h_{f}=\sqrt{\Delta_{y}}$ at $\theta=\arccos h_{f}$; and an energy per site $\varepsilon=\left(1+\Delta_{y}\right) / 4$. These results justify rigorously recent numerical findings [13].

Comments.- We have introduced a simple and general analytic approach to the exact determination of factorized ground states in quantum spin systems. We have applied the scheme to spin- $1 / 2$ models with general anisotropic Heisenberg-like interactions of arbitrary range and for lattices of arbitrary dimensions. Besides the rigorous derivation of the few known, mainly numerical, results, we have showed that our method allows to determine exactly novel classes of factorization points in various models, generally non-exactly solvable, for different lattice dimensions and for different interaction ranges. These novel exact solutions of non exactly solvable models are obtained for nontrivial sets of values of the Hamiltonian parameters. Furthermore, according to the general theorem by Kurmann et al. on factorization [5], given any Hamiltonian of the form Eq. (1) with generic spin $S>1 / 2$, the ground state of the system is factorized at the same value of the external field $h=h_{f}$ [Eq. (8)], at which factorization occurs in the corresponding spin- $1 / 2$ model. Therefore, the method and the results derived in the present paper are straightforwardly generalized to interacting systems with arbitrary value of the spin which are endowed with the same Hamiltonian structures as in the spin- $1 / 2$ case. Further applications to other systems, defined, e.g., on ladders and coupled planes, or to models with frustration and in complex geometries, can be in principle carried out by suitably adapting and specializing the general framework introduced in the present work. From a conceptual standpoint, the method realizes a rigorous and analytic implementation of concepts motivated by quantum information theory to obtain genuinely new insights on founding open questions of condensed matter physics.

Acknowledgements.- We thank T. Roscilde and P. Verrucchi for useful discussions. We acknowledge financial support from MIUR under PRIN National Project 2005 and from CNR-INFM Coherentia.

*Corresponding author: illuminati@sa.infn.it
[1] Y. Makhlin, G. Schön, and A. Shnirman, Rev. Mod. Phys. 73, 357 (2001); V. Giovannetti, S. Lloyd, and L. Maccone, Science 306, 1330 (2004); F. Dell'Anno, S. De Siena, and F. Illuminati, Phys. Rep. 428, 53 (2006).

[2] M. J. Hartmann, F. G. S. L. Brandão, and M. B. Plenio, Phys. Rev. Lett. 99, 160501 (2007); D. G. Angelakis, M. F. Santos, and S. Bose, Phys. Rev. A 76, 031805(R) (2007); A. Kay and D. G. Angelakis, arXiv:0802.0488

[3] A. Sørensen and K. Mølmer, Phys. Rev. Lett. 83, 2274 (1999); A. B. Kuklov and B. V. Svistunov, Phys. Rev. Lett. 90, 100401 (2003); L.-M. Duan, E. Demler, and M. D. Lukin, Phys. Rev. Lett. 91, 090402 (2003).

[4] L. Amico, R. Fazio, A. Osterloh, and V. Vedral, arXiv:quant-ph/0703044 and Rev. Mod. Phys., to appear.

[5] J. Kurmann, H. Thomas, and G. Müller, Physica A (Amsterdam) 112, 235 (1982).

[6] T. Roscilde, P. Verrucchi, A. Fubini, S. Haas, and V. Tognetti, Phys. Rev. Lett. 93, 167203 (2004); ibid. 94, 147208 (2005).

[7] V. Kendon, K. Nemoto, and W. J. Munro, J. Mod. Opt. 49, 1709 (2002); R. Oliveira, O. C. O. Dahlsten, and M. B. Plenio, Phys. Rev. Lett. 98, 130502 (2007).

[8] L. Amico, F. Baroni, A. Fubini, D. Patané, V. Tognetti, and P Verrucchi, Phys. Rev. A 74, 022322 (2006); F. Baroni, A. Fubini, V. Tognetti, and P. Verrucchi, J. Phys. A 40, 9845 (2007).

[9] S. Bose, Phys. Rev. Lett. 91, 207901 (2003); Contemporary Physics 48, 13 (2007).

[10] V. Coffman, J. Kundu, and W. K. Wootters, Phys. Rev. A 61, 052306 (2000); T. J. Osborne and F. Verstraete, Phys. Rev. Lett. 96, 220503 (2006).

[11] S. M. Giampaolo and F. Illuminati, Phys. Rev. A 76, 042301 (2007).

[12] S. M. Giampaolo, F. Illuminati, P. Verrucchi, and S. De Siena, Phys. Rev. A 77, 012319 (2008).

[13] S. Dusuel and J. Vidal, Phys. Rev. B 71, 224420 (2005).

[14] If one does not require the vanishing of the EXE, $\theta$ and the ground state Eq. (5) are expressed as a simple function of the magnetizations $M_{\alpha}$ 's alone. Imposing the conditions under which Eq. 5] is an eigenstate of the Hamiltonian, one merely obtains a relation between $h_{f}$ and the $M_{\alpha}$ 's. If the analytic expression of at least one of the $M_{\alpha}$ 's is known, it is possible to obtain an expression of the factorization point $h_{f}$ as a function of the Hamiltonian parameters. But this occurs only in few special cases.

[15] Notice that while Eq. (7) involves only the couplings at a fixed distance $r$, Eq. (4) and Eq. (8) involve the net interactions. Hence, in models with short range interactions $\left(J_{\alpha}^{r}=\right.$ $0 \forall \alpha, r \geq 2$ ), if Eq. (4) and Eq. (8) have two real solutions, then Eqs. (7) is satisfied $\forall r$. On the contrary, for models with finite and long range interactions there may be cases when Eq. (4) and Eq. (8) have two real solutions but Eqs. (7) are not verified $\forall r$ and thus factorization cannot occur.

[16] H. J. Lipkin, N. Meshkov, and A. J. Glick, Nucl. Phys. 62, 188 (1965); ibid. 62, 199 (1965); ibid. 62, 211 (1965). 